\title{
Cancer cachexia
}

\section{Marcus E Martignoni, Philipp Kunze and Helmut Friess*}

\author{
Address: Department of General Surgery, University of Heidelberg, Im Neuenheimer Feld 110 Germany \\ Email: Marcus E Martignoni - me.martignoni@med.uni-heidelberg.de; Philipp Kunze - philipp_kunze@med.uni-heidelberg.de; \\ Helmut Friess* - helmut_friess@med.uni-heidelberg.de \\ * Corresponding author
}

Published: 05 November 2003

Molecular Cancer 2003, 2:36

This article is available from: http://www.molecular-cancer.com/content/2/I/36

(c) 2003 Martignoni et al; licensee BioMed Central Ltd. This is an Open Access article: verbatim copying and redistribution of this article are permitted in all media for any purpose, provided this notice is preserved along with the article's original URL.
Received: 10 October 2003

Accepted: 05 November 2003

\begin{abstract}
In recent years many efforts of researchers and clinicians were made to improve our knowledge of cachexia syndrome. Not only cancer, but also many chronic or end-stage diseases such as AIDS, chronic obstructive pulmonary disease (COPD), rheumatoid arthritis, tuberculosis and Crohn's disease are associated with cachexia, a condition of abnormally low weight, weakness, and general bodily decline which deteriorates quality of life and reduces the prognosis of the patients who suffer from it. In the present editorial we will focus cachexia related on cancer and provide some insight into this prognosis-limiting syndrome.
\end{abstract}

\section{Editorial}

Cancer cachexia occurs most frequently in malignancy and is associated with more than $20 \%$ of cancer deaths [1]. Patients with upper gastrointestinal cancer are especially likely to suffer from substantial weight loss, and patients with pancreatic cancer have the highest frequency of developing a cachectic syndrome. Thus the research groups and physicians dealing with pancreatic cancer are very interested in finding an effective treatment for cachectic patients. But there is still little known about this clinical issue, and our knowledge grows slowly. Much more research and many more clinical trials are needed to increase our understanding of the syndrome and to develop therapeutic strategies for one of the major symptoms of cancer.

The word "cachexia" comes from the Greek words "kakos" and "hexis", meaning "bad conditions" [2]. Cachexia is a complex metabolic status with progressive weight loss and depletion of host reserves of adipose tissue and skeletal muscle. Cachexia should be suspected if involuntary weight loss of greater than five percent of premorbid weight occurs within a six-month period [3]. Cachexia represents the clinical consequence of a chronic, systemic inflammatory response, with high hepatic synthesis of acute-phase proteins resulting in depletion of essential amino acids [4]. In contrast, in starvation only fat metabolism is increased while the organism tries to conserve lean body mass [5].

In addition to metabolic changes, cachexia is often associated with anorexia. In cancer patients there can be mechanical interference such as obstructions, as well as treatment-related toxicity. In patients receiving chemotherapy or radiation, subsequent nausea, vomiting and diarrhea can contribute to weight loss. But the lack of nutrients alone cannot explain the metabolic changes seen in cachexia. In clinical trials, nutritional supplementation and dietary counseling failed to increase body weight [6]. Several appetite-stimulating drugs have been tested in an attempt to increase the food intake of cachexia 
patients, but most of them had little or no effect on body weight [7].

Only limited treatment options exist for patients with clinical cancer cachexia. In one trial, corticosteroids improved the sensation of well-being and led to increased food intake, but this effect lasted only a few weeks [2]. Progestogens such as megestrol acetate and medroxyprogesterone acetate also failed to meet expectations $[3,8]$. Body composition analysis showed that the weight gain resulted only from increased body fat and fluid, with no change in lean body mass [2]. Additionally, therapy with progestogens led to a decline in the response rate to chemotherapy and an increase in the frequency of thrombembolic events $[3,7]$.

Much research is currently focused on determining the mechanism of the development of cachexia. There are two main theories of the development of cancer cachexia.

The first theory is the pathological alteration of control cycles. Food-intake is regulated through a complex system of hormones and neuropeptides. Inui et al. demonstrated that Neuropeptid Y (NPY), the most potent feeding-stimulatory peptide in this cycle, is deregulated in the hypothalamic orexigenic network, leading to decreased energy intake but high metabolic demand for nutrients [9]. High levels of leptin, a hormone secreted by adipocytes, block the release of NPY. In cachexia the leptin feedback loop seems to become out of control, altering the neuropeptidergic control cycles [9].

The second theory is based on the idea that tumor-derived factors maintain the cachectic syndrome. Tisdale et al. postulated a factor that was extracted from the urine of cachectic patients and which induces protein degradation in skeletal muscle by upregulation of the ubiquitin-proteasome pathway. This proteolysis-inducing factor (PIF) is closely related to weight loss in cachexia, and in a recent study it was shown that PIF is produced in human colon cancer $[1,10,11]$.

A second factor extracted from the urine of cachectic patients - lipid mobilizing factor (LMF) - is closely related to weight loss and induces lipolysis in murine adipocytes. A recent study showed that this lipolytic process is mediated through the $\beta-3$ adrenoceptors. LMF produces a significant increase in the UCPs in brown adipose tissue, skeletal muscle and liver [12].

Mitochondrial uncoupling proteins (UCPs) 1, 2, and 3 are involved in the control of energy metabolism through thermogenesis in brown adipose tissue and possibly in skeletal muscle tissue in humans. In many animal models, overexpression of UCPs (especially UCP 2) in white adipocytes and in muscle and liver tissue was associated with cachexia [6].

Both theories contribute to a better understanding of the development of cancer cachexia. However, it is still uncertain how they interact and whether they come into play at the beginning or at the end stage of the disease.

Despite the controversial discussion of cachexia-inducing mechanisms uncertainty over what causes cachexia, it is quite clear that proinflammatory cytokines are linked to all pathways that induce cachexia. As mentioned, cachexia is associated with a chronic systemic inflammatory response and the elevation of acute phase proteins. High serum levels of IL-1, IL- 6 and INF gamma are present in many cancer patients, and the levels of these cytokines seem to correlate with tumor progression.

These cytokines stimulate the expression of leptin and/or mimic the hypothalamic effect of negative feedback from leptin by disarranging the signaling pathway of NPY, resulting in long-term inhibition of food intake. IL-1 antagonizes NPY - induced feeding in rats and disrupts the orexigenic pathway of NPY. On the other hand, central corticotropin-releasing factor (CRF), which is upregulated by IL-1, seems to influence satiety, and is a potent anorexigenic signal [13].

UCP expression is not only increased by LMF. Tumor necrosis factor alpha (TNF- $\alpha$ ) also increases the mRNA levels of UCP2 and 3. In combination with INF $\gamma$ TNF- $\alpha$ activates the transcription factor NFKB that leads to reduction of Myo D, a transcription factor essential for repairing damaged muscle tissue [14].

Although IL-6 is one of the key cytokines involved in the development of cachexia, the definite mechanisms have not yet been clarified. Improvements in appetite and weight gain through decreased cytokine expression after the application of corticosteroids or special antagonists like IL- 6 antibodies were seen over short periods, but further investigations of the cytokine system are necessary to elucidate the interaction between host and tumor-derived cytokines and to determine their effect on biochemical mechanisms.

\section{Conclusions}

Many trials have been performed in the search for a treatment for cachexia, but most therapies have not fulfilled expectations. Currently, eicosapaentanoic acid is being tested in cachectic patients. Eicosapaentanoic acid seems to interfere with the signaling pathway of PIF, and first results are promising [4]. Future therapies may consist of anticatabolic and anabolic drugs in combination with appetite stimulants. 
Although in recent years our understanding of cachexia has increased, we are still in the fledgling stages. The scientists and clinicians dedicated to finding an effective treatment for cachectic patients have their work cut out for them.

\section{Author's contribution}

MEM and PK drafted the paper. HF provided comments and suggestions for its finalization. All authors read and approved the final manuscript.

\section{References}

I. Tisdale MJ: Cachexia in cancer patients. Nat Rev Cancer 2002, 2:862-87I.

2. Tisdale MJ: Biology of cachexia. J Natl Cancer Inst 1997, 89:1763-1773.

3. Inui A: Cancer anorexia-cachexia syndrome: current issues in research and management. CA Cancer J Clin 2002, 52:72-91.

4. Wigmore SJ, Plester CE, Richardson RA and Fearon KC: Changes in nutritional status associated with unresectable pancreatic cancer. BrJ Cancer 1997, 75: 106-109.

5. Tisdale MJ: Cancer anorexia and cachexia. Nutrition 200I, 17:438-442.

6. Kotler DP: Cachexia. Ann Intern Med 2000, 133:622-634.

7. Gagnon $B$ and Bruera $E:$ A review of the drug treatment of cachexia associated with cancer. Drugs 1998, 55:675-688.

8. Loprinzi CL, Schaid DJ, Dose AM, Burnham NL and Jensen MD: Body-composition changes in patients who gain weight while receiving megestrol acetate. J Clin Oncol 1993, I I:152-154.

9. Inui A: Cancer anorexia-cachexia syndrome: are neuropeptides the key? Cancer Res 1999, 59:4493-450I.

10. Tisdale MJ: The 'cancer cachectic factor'. Support Care Cancer 2003, II:73-78

II. Cabal-Manzano R, Bhargava P, Torres-Duarte A, Marshall J, Bhargava $P$ and Wainer IW: Proteolysis-inducing factor is expressed in tumours of patients with gastrointestinal cancers and correlates with weight loss. Br J Cancer 200I, 84:I599-I60I.

12. Islam-Ali BS and Tisdale MJ: Effect of a tumour-produced lipidmobilizing factor on protein synthesis and degradation. $\mathrm{Br} J$ Cancer 200I, 84: 1648-1655.

13. Bruera $\mathrm{E}: \mathrm{ABC}$ of palliative care. Anorexia, cachexia, and nutrition. $B M]$ | 1997, 3 15:1219-1222.

14. Guttridge DC, Mayo MW, Madrid LV, Wang CY and Baldwin A.S.Jr.: NF-kappaB-induced loss of MyoD messenger RNA: possible role in muscle decay and cachexia. Science 2000, 289:2363-2366.
Publish with Bio Med Central and every scientist can read your work free of charge

"BioMed Central will be the most significant development for disseminating the results of biomedical research in our lifetime. "

Sir Paul Nurse, Cancer Research UK

Your research papers will be:

- available free of charge to the entire biomedical community

- peer reviewed and published immediately upon acceptance

- cited in PubMed and archived on PubMed Central

- yours - you keep the copyright 